\title{
An infinite family of higher-order difference operators that commute with Ruijsenaars operators of type $A$
}

\author{
Masatoshi Noumi ${ }^{1}$ (D) Ayako Sano ${ }^{2}$
}

Received: 29 March 2021 / Revised: 21 June 2021 / Accepted: 23 June 2021 / Published online: 7 July 2021

(C) The Author(s) 2021

\section{Abstract}

We introduce a new infinite family of higher-order difference operators that commute with the elliptic Ruijsenaars difference operators of type $A$. These operators are related to Ruijsenaars' operators through a formula of Wronski type.

Keywords Ruijsenaars operators · Wronski formula $\cdot$ Macdonald polynomials

Mathematics Subject Classification 81R12 $\cdot 33 \mathrm{D} 67$

\section{Introduction}

In Ruijsenaars's study of relativistic quantum integrable systems [11,12], he introduced a commuting family of linear difference operators in $n$ variables, denoted by $D_{1}, \ldots, D_{n}$, involving sigma functions as coefficients. In this paper, we construct an explicit infinite family of difference operators $H_{0}, H_{1}, H_{2}, \ldots$ in the commutative algebra $\mathbb{C}\left[D_{1}, \ldots, D_{n}\right]$ which are related to $D_{r}(r=1, \ldots, n)$ through a formula of Wronski type. This construction is applicable also to difference operators with trigonometric and rational coefficients. In order to deal with the elliptic, trigonometric and rational cases simultaneously, as in [5,6] we formulate our results in terms of an entire function $[z]$ satisfying the three-term relation (1.1).

Masatoshi Noumi: On leave from: Department of Mathematics, Kobe University, Rokko, Kobe 657-8501, Japan.

$\bowtie \quad$ Masatoshi Noumi

noumi@kth.se; noumi@math.kobe-u.ac.jp

1 Department of Mathematics, KTH Royal Institute of Technology, SE-100 44 Stockholm, Sweden

2 Department of Mathematics, Kobe University (graduate), Rokko, Kobe 657-8501, Japan 
We fix a nonzero entire function $[z]$ in one complex variable $z \in \mathbb{C}$ satisfying the three-term relation of Hirota type

$$
[z \pm \alpha][\beta \pm \gamma]+[z \pm \beta][\gamma \pm \alpha]+[z \pm \gamma][\alpha \pm \beta]=0
$$

for any $\alpha, \beta, \gamma \in \mathbb{C}$, where $[\alpha \pm \beta]=[\alpha+\beta][\alpha-\beta]$. We remark that a generic solution of this functional equation is given by

$$
[z]=\text { const. } e^{c z^{2}} \sigma(z ; \Omega) \quad(c \in \mathbb{C}),
$$

where $\sigma(z ; \Omega)$ denotes the Weierstrass sigma function associated with a period lattice $\Omega=\mathbb{Z} \omega_{1} \oplus \mathbb{Z} \omega_{2}$. It is also satisfied by the functions

$$
[z]=\text { const. } e^{c z^{2}} \sin (\pi z / \omega), \quad[z]=\text { const. } e^{c z^{2}} z,
$$

which are trigonometric and rational degenerations of the generic solution above. It is known that any solution of (1.1) belongs to one of these three categories. Throughout this paper, we denote by $D_{r}=D_{r}^{x}(r=1, \ldots, n)$ the Ruijsenaars operators in $n$ variables $x=\left(x_{1}, \ldots, x_{n}\right)$ with parameters $(\delta, \kappa)$, associated with $[z]$. They are defined by

$$
D_{r}^{x}=\sum_{I \subseteq\{1, \ldots, n\} ;|I|=r} \prod_{i \in I ; j \notin I} \frac{\left[x_{i}-x_{j}+\kappa\right]}{\left[x_{i}-x_{j}\right]} \prod_{i \in I} T_{x_{i}}^{\delta},
$$

where $I$ runs over all subsets of indices of cardinality $r$ and $T_{x_{i}}^{\delta}$ stands for the $\delta$-shift operator $x_{i} \rightarrow x_{i}+\delta$ in $x_{i}$ for each $i=1, \ldots, n$. It is proved by Ruijsenaars [11] that these operators $D_{r}$ commute with each other, namely

$$
D_{r} D_{s}=D_{s} D_{r} \quad \text { for all } r, s=1, \ldots, n,
$$

on the basis of a certain functional identity for the sigma function. We denote by $\mathcal{R}=\mathbb{C}\left[D_{1}, \ldots, D_{n}\right]$ the commutative algebra generated by $D_{r}(r=1, \ldots, n)$ and refer to it as the commutative algebra of Ruijsenaars operators (of type $A_{n-1}$ ). We also define $D_{0}=1$, and $D_{r}=0$ for $r>n$. Note also that these operators $D_{r}$ $(r=1, \ldots, n)$ in the trigonometric case are the Macdonald $q$-difference operators expressed additively.

We define an infinite family of difference operators $H_{l}=H_{l}^{x}(l=0,1,2 \ldots)$ by

$$
H_{l}^{x}=\sum_{\mu_{1}+\cdots+\mu_{n}=l} \prod_{1 \leq i<j \leq n} \frac{\left[x_{i}-x_{j}+\left(\mu_{i}-\mu_{j}\right) \delta\right]}{\left[x_{i}-x_{j}\right]} \prod_{i=1}^{n} \prod_{j=1}^{n} \prod_{k=0}^{\mu_{i}-1} \frac{\left[x_{i}-x_{j}+\kappa+k \delta\right]}{\left[x_{i}-x_{j}+\delta+k \delta\right]} \prod_{i=1}^{n} T_{x_{i}}^{\mu_{i} \delta}
$$

These operators are expressed briefly as

$$
H_{l}^{x}=\sum_{\mu \in \mathbb{N}^{n} ;|\mu|=l} \frac{\Delta(x+\mu \delta)}{\Delta(x)} \prod_{i, j=1}^{n} \frac{\left[x_{i}-x_{j}+\kappa\right]_{\mu_{i}}}{\left[x_{i}-x_{j}+\delta\right]_{\mu_{i}}} T_{x}^{\mu \delta}
$$


in terms of the difference product $\Delta(x)=\prod_{1 \leq i<j \leq n}\left[x_{i}-x_{j}\right]$, and the $\delta$-shifted factorials

$$
[z]_{k}=[z][z+\delta] \cdots[z+(k-1) \delta] \quad(k=0,1,2, \ldots)
$$

for $[z]$. Also, for each multi-index $\mu=\left(\mu_{1}, \ldots, \mu_{n}\right) \in \mathbb{N}^{n}, \mathbb{N}=\{0,1,2, \ldots\}$, we define $|\mu|=\mu_{1}+\cdots+\mu_{n}$ and $T_{x}^{\mu \delta}=T_{x_{1}}^{\mu_{1} \delta} \cdots T_{x_{n}}^{\mu_{n} \delta}$.

Theorem 1.1 The linear difference operators $H_{l}=H_{l}^{x}(l=0,1,2, \ldots)$ defined as above belong to the commutative algebra $\mathcal{R}=\mathbb{C}\left[D_{1}, \ldots, D_{n}\right]$ of Ruijsenaars operators. In particular, one has

$$
D_{r} H_{s}=H_{s} D_{r}, \quad H_{r} H_{s}=H_{s} H_{r} \quad(r, s=0,1,2, \ldots) .
$$

We first remark that the family of difference operators $H_{l}(l=0,1,2, \ldots)$ originates from the kernel identities for Ruijsenaars operators ([6]) and the duality transformation formulas for multiple elliptic hypergeometric series ([5,10]). Let $G(z)$ be a nonzero meromorphic function on $\mathbb{C}$ such that $G(z+\delta)=[z] G(z)$, and define the kernel function $\Phi(x ; y)$ of Cauchy type by

$$
\Phi(x ; y)=\prod_{i=1}^{n} \prod_{k=1}^{n} \frac{G\left(x_{i}+y_{k}\right)}{G\left(x_{i}+y_{k}+\kappa\right)}
$$

for $x=\left(x_{1}, \ldots, x_{n}\right)$ and $y=\left(y_{1}, \ldots, y_{n}\right)$. Then, from [5], Theorem 1.3, we have the kernel identity

$$
D_{r}^{x} \Phi(x ; y)=D_{r}^{y} \Phi(x ; y) \quad(r=0,1, \ldots, n)
$$

for the Ruijsenaars operators $D_{r}(r=0,1, \ldots, r)$. On the other hand, the duality transformation formula for multiple elliptic hypergeometric series implies

$$
\begin{aligned}
& \sum_{\mu \in \mathbb{N}^{m} ;|\mu|=r} \frac{\Delta(x+\mu \delta)}{\Delta(x)} \prod_{i, j=1}^{n} \frac{\left[x_{i}-x_{j}+\kappa\right]_{\mu_{i}}}{\left[x_{i}-x_{j}+\delta\right]_{\mu_{i}}} \prod_{i, k=1}^{n} \frac{\left[x_{i}+y_{k}\right]_{\mu_{i}}}{\left[x_{i}+y_{k}+\kappa\right]_{\mu_{i}}} \\
= & \sum_{\nu \in \mathbb{N}^{m} ;|\nu|=r} \frac{\Delta(y+\nu \delta)}{\Delta(y)} \prod_{k, l=1}^{n} \frac{\left[y_{k}-y_{l}+\kappa\right]_{\nu_{k}}}{\left[y_{k}-y_{l}+\delta\right]_{v_{k}}} \prod_{k, i=1}^{n} \frac{\left[y_{k}+x_{i}\right]_{\nu_{k}}}{\left[y_{k}+x_{i}+\kappa\right]_{\nu_{k}}} \quad(r=0,1,2, \ldots),
\end{aligned}
$$

as the special case where $m=n$ and $a_{i}=b_{i}=\kappa(i=1, \ldots, n)$ in the notation of [5], Theorem 2.2. This means that

$$
H_{r}^{x} \Phi(x ; y)=H_{r}^{y} \Phi(x ; y) \quad(r=0,1,2, \ldots) .
$$

Namely, the kernel function for the Ruijsenaars operators $D_{r}(r=0,1, \ldots, n)$ simultaneously intertwines the operators $H_{r}(r=0,1,2 \ldots)$. In view of this fact, it would be reasonable to expect that the operators $H_{r}$ should already belong to the commutative algebra $\mathbb{C}\left[D_{1}, \ldots, D_{n}\right]$ of Ruijsenaars operators. Theorem 1.1 ensures that it is actually the case. 
In this paper, we prove Theorem 1.1 as a consequence of the following recurrence formula of Wronski type for $H_{l}(l=0,1,2, \ldots)$.

Theorem 1.2 The difference operators $H_{l}(l=0,1,2, \ldots)$ satisfy the following recurrence formula in relation to $D_{r}(r=1, \ldots, n)$ :

$$
\sum_{r+s=l}(-1)^{r}[r \kappa+s \delta] D_{r} H_{s}=0 \quad(l=1,2, \ldots) .
$$

Recall that the elementary symmetric functions

$$
e_{r}=e_{r}(\xi)=\sum_{1 \leq i_{1}<\cdots<i_{r} \leq m} \xi_{i_{1}} \cdots \xi_{i_{r}} \quad(r=0,1,2, \ldots)
$$

in $\xi=\left(\xi_{1}, \ldots, \xi_{n}\right)$, and the complete homogeneous symmetric functions

$$
h_{l}=h_{l}(\xi)=\sum_{\mu_{1}+\cdots+\mu_{n}=l} \xi_{1}^{\mu_{1}} \ldots \xi_{n}^{\mu_{n}} \quad(l=0,1,2, \ldots)
$$

are related to each other through the Wronski formula ${ }^{1}$

$$
\sum_{r+s=l}(-1)^{r} e_{r} h_{s}=0 \quad(l=1,2, \ldots) .
$$

See [7]. Theorem 1.2 can be thought of as an operator version of this Wronski formula for symmetric functions. A proof of Theorem 1.2 will be given in Sect. 2 by using a functional identity for [z] (Lemma 2.3).

From the recurrence formulas

$$
\begin{aligned}
& {[\delta] H_{1}-[\kappa] D_{1}=0,} \\
& {[2 \delta] H_{2}-[\kappa+\delta] D_{1} H_{1}+[2 \kappa] D_{2}=0,} \\
& {[3 \delta] H_{3}-[\kappa+2 \delta] D_{1} H_{2}+[2 \kappa+\delta] D_{2} H_{1}-[3 \kappa] D_{3}=0,}
\end{aligned}
$$

we see inductively that $H_{l}$ belongs to the commutative algebra $\mathcal{R}=\mathbb{C}\left[D_{1}, \ldots, D_{n}\right]$ of Ruijsenaars operators for all $l=0,1,2, \ldots$ In fact, we have

$$
\begin{aligned}
& H_{1}=\frac{[\kappa]}{[\delta]} D_{1}, \\
& H_{2}=\frac{[\kappa][\kappa+\delta]}{[\delta][2 \delta]} D_{1}^{2}-\frac{[2 \kappa]}{[2 \delta]} D_{2}, \\
& H_{3}=\frac{[\kappa][\kappa+\delta][\kappa+2 \delta]}{[\delta][2 \delta][3 \delta]} D_{1}^{3}-\frac{[2 \kappa][\kappa+2 \delta]}{[2 \delta][3 \delta]} D_{2} D_{1}-\frac{[\kappa][2 \kappa+\delta]}{[\delta][3 \delta]} D_{1} D_{2}+\frac{[3 \kappa]}{[3 \delta]} D_{3},
\end{aligned}
$$

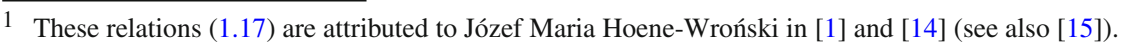


The relationship between the two families of difference operators $D_{r}(r=01,2, \ldots)$ and $H_{l}(l=0,1,2, \ldots)$ is described in the following.

Proposition 1.3 For each $l=0,1,2, \ldots$, the difference operator $H_{l}$ is expressed in terms of $D_{r}(r=0,1, \ldots)$ by the determinant formula

$$
H_{l}=\operatorname{det}\left(\frac{[(i-j+1) \kappa+(j-1) \delta]}{[i \delta]} D_{i-j+1}\right)_{i, j=1}^{l} \quad(l=0,1,2, \ldots) .
$$

Conversely,

$$
D_{l}=\operatorname{det}\left(\frac{[(i-j+1) \delta+(j-1) \kappa]}{[i \kappa]} H_{i-j+1}\right)_{i, j=1}^{l} \quad(l=0,1,2, \ldots) .
$$

Proposition 1.4 For each $l=1,2, \ldots$, the difference operator $H_{l}$ is expressed explicitly as

$$
H_{l}=\sum_{d=1}^{l}(-1)^{l-d} \sum_{r_{1}+\cdots+r_{d}=l ; r_{i} \geq 1}\left(\prod_{i=1}^{d} \frac{\left[\left(r_{1}+\cdots+r_{i-1}\right) \delta+r_{i} \kappa\right]}{\left[\left(r_{1}+\cdots+r_{i}\right) \delta\right]}\right) D_{r_{1}} \cdots D_{r_{d}}
$$

in terms of $D_{r}(r=0,1, \ldots)$.

We also summarize the kernel identities relevant to the difference operators $D_{r}$ and $H_{r}$ for the sake of reference.

Theorem 1.5 (1) For two sets of variables $x=\left(x_{1}, \ldots, x_{n}\right)$ and $y=\left(y_{1}, \ldots, y_{n}\right)$, the kernel function $\Phi(x, y)$ of Cauchy type in (1.10) satisfies the following two types of kernel identities:

$$
\begin{array}{lll}
(D D) & D_{r}^{x} \Phi(x ; y)=D_{r}^{y} \Phi(x ; y) & (r=0,1, \ldots, n), \\
(H H) & H_{r}^{x} \Phi(x ; y)=H_{r}^{y} \Phi(x ; y) & (r=0,1,2, \ldots) .
\end{array}
$$

(2) For two sets of variables $x=\left(x_{1}, \ldots, x_{m}\right)$ and $y=\left(y_{1}, \ldots, y_{n}\right)$, let

$$
\Psi(x ; y)=\prod_{i=1}^{m} \prod_{k=1}^{n}\left[x_{i}-y_{k}\right]
$$

be the kernel function of dual Cauchy type. Under the balancing condition $m \kappa+n \delta=$ $0, \Psi(x ; y)$ satisfies the kernel identity

$(H D) \quad H_{r}^{x} \Psi(x ; y)=(-1)^{r} \widehat{D}_{r}^{y} \Psi(x ; y) \quad(r=0,1,2, \ldots)$,

where $\widehat{D}_{r}^{y}$ denotes the difference operator obtained from $D_{r}^{y}$ by exchanging the parameters $\delta$ and $\kappa$. 
Propositions 1.3 and 1.4 are consequences of the recurrence formula of Wronski type (see Sect. 3). After a complement on kernel identities for the Ruijsenaars operators (Sect. 4), we finally give some remarks on the trigonometric case in Sect. 5.

Notes: This paper is based on a collaboration of the authors which was completed as master's thesis [13] of the second author in Japanese. Also, an earlier version of the present paper, written around 2012, has been circulated among some researchers. For these reasons, some of the results in this paper are already cited in several studies $[2,3,8,9]$ with reference to a private communication or to a paper in preparation.

\section{Recurrence formula of Wronski type}

In this section, we give a proof of Theorem 1.2. Our goal is to establish the recurrence formula of Wronski type between the two sequences of difference operators $D_{r}(r=$ $0,1,2, \ldots)$ and $H_{s}(s=0,1,2, \ldots)$.

Theorem 2.1 The difference operators $H_{l}(l=0,1,2 \ldots)$ defined by (1.6) satisfy the recurrence formulas

$$
\sum_{r+s=l}(-1)^{r}[r \kappa+s \delta] D_{r} H_{s}=0 \quad(l=1,2, \ldots)
$$

Since $D_{0}=1$, by this theorem we see inductively that $H_{l}$ belong to $\mathbb{C}\left[D_{1}, \ldots, D_{n}\right]$ for all $l=0,1,2, \ldots$.

Theorem 2.2 The difference operators $H_{l}(l=0,1,2 \ldots)$ belong to the commutative algebra $\mathbb{C}\left[D_{1}, \ldots, D_{n}\right]$ of Ruijsenaars operators. In particular, one has

$$
D_{r} H_{s}=H_{s} D_{r}, \quad H_{r} H_{s}=H_{s} H_{r} \quad(r, s=0,1,2, \ldots) .
$$

Proof of Theorem 2.1 We express the difference operators $D_{r}$ as

$$
D_{r}=\sum_{|I|=r} A_{I}(x) T_{x}^{\epsilon_{I} \delta}, \quad A_{I}(x)=\prod_{i \in I ; j \notin I} \frac{\left[x_{i}-x_{j}+\kappa\right]}{\left[x_{i}-x_{j}\right]}=\frac{\Delta\left(x+\epsilon_{I} \kappa\right)}{\Delta(x)}
$$

where we define $\epsilon_{I}=\sum_{i \in I} \epsilon_{i}$ by using the unit vectors $\epsilon_{1}, \ldots, \epsilon_{n}$ of $\mathbb{N}^{n}$. Similarly, we express $H_{l}$ as

$$
H_{l}=\sum_{|\mu|=l} H_{\mu}(x) T_{x}^{\mu \delta}, \quad H_{\mu}(x)=\frac{\Delta(x+\mu \delta)}{\Delta(x)} \prod_{i, j=1}^{n} \frac{\left[x_{j}-x_{i}+\kappa\right]_{\mu_{j}}}{\left[x_{j}-x_{i}+\delta\right]_{\mu_{j}}} .
$$


When $r+s=l$, we compute

$$
\begin{aligned}
D_{r} H_{s} & =\sum_{|I|=r} \sum_{|\mu|=s} A_{I}(x) H_{\mu}\left(x+\epsilon_{I} \delta\right) T_{x}^{\left(\epsilon_{I}+\mu\right) \delta} \\
& =\sum_{|\lambda|=l} \sum_{I \subseteq \operatorname{supp}(\lambda) ;|I|=r} A_{I}(x) H_{\lambda-\epsilon_{I}}\left(x+\epsilon_{I} \delta\right) T_{x}^{\lambda \delta},
\end{aligned}
$$

where $\operatorname{supp}(\lambda)=\left\{i \in\{1, \ldots, n\} \mid \lambda_{i}>0\right\}$. Hence, the recurrence formula (2.1) is equivalent to

$$
\sum_{|\lambda|=l}\left(\sum_{I \subseteq \operatorname{supp}(\lambda)}(-1)^{|I|}[|I| \kappa+(|\lambda|-|I|) \delta] A_{I}(x) H_{\lambda-\epsilon_{I}}\left(x+\epsilon_{I} \delta\right)\right) T_{x}^{\lambda \delta}=0
$$

saying that

$$
\sum_{I \subseteq \operatorname{supp}(\lambda)}(-1)^{|I|}[|I| \kappa+(|\lambda|-|I|) \delta] A_{I}(x) H_{\lambda-\epsilon_{I}}\left(x+\epsilon_{I} \delta\right)=0
$$

for any $\lambda \in \mathbb{N}^{n}$ with $|\lambda|>0$. We now make the expression $A_{I}(x) H_{\lambda-\epsilon_{I}}\left(x+\epsilon_{I} \delta\right)$ explicit. Setting $L=\operatorname{supp}(\lambda)$, we have

$$
\begin{aligned}
A_{I}(x) H_{\lambda-\epsilon_{I}}\left(x+\epsilon_{I} \delta\right)= & \frac{\Delta\left(x+\epsilon_{I} \kappa\right)}{\Delta(x)} \frac{\Delta(x+\lambda \delta)}{\Delta\left(x+\epsilon_{I} \delta\right)} \\
& \cdot \prod_{i \in I ; j \in I} \frac{\left[x_{j}-x_{i}+\kappa\right] \lambda_{j}-1}{\left[x_{j}-x_{i}+\delta\right]_{\lambda_{j}-1}} \prod_{i \in I ; j \in L \backslash I} \frac{\left[x_{j}-x_{i}+\kappa-\delta\right]_{\lambda_{j}}}{\left[x_{j}-x_{i}\right]_{\lambda_{j}}} \\
& \cdot \prod_{i \notin I ; j \in I} \frac{\left[x_{j}-x_{i}+\kappa+\delta\right]_{j}-1}{\left[x_{j}-x_{i}+2 \delta\right]_{\lambda_{j}-1}} \prod_{i \notin I ; j \in L \backslash I} \frac{\left[x_{j}-x_{i}+\kappa\right]_{j}}{\left[x_{j}-x_{i}+\delta\right]_{\lambda_{j}}} .
\end{aligned}
$$

Noting that

$$
\begin{gathered}
\frac{\Delta\left(x+\epsilon_{I} \kappa\right)}{\Delta(x)} \frac{\Delta(x+\lambda \delta)}{\Delta\left(x+\epsilon_{I} \delta\right)}=\frac{\Delta(x+\lambda \delta)}{\Delta(x)} \frac{\Delta\left(x+\epsilon_{I} \kappa\right)}{\Delta\left(x+\epsilon_{I} \delta\right)} \\
=\frac{\Delta(x+\lambda \delta)}{\Delta(x)} \prod_{i \notin I ; j \in I} \frac{\left[x_{j}-x_{i}+\kappa\right]}{\left[x_{j}-x_{i}+\delta\right]}
\end{gathered}
$$

we can compute $A_{I}(x) H_{\lambda-\epsilon_{I}}\left(x+\epsilon_{I} \delta\right)$ as follows:

$$
\begin{aligned}
A_{I}(x) H_{\lambda-\epsilon_{I}}\left(x+\epsilon_{I} \delta\right)= & \frac{\Delta(x+\lambda \delta)}{\Delta(x)} \prod_{i \notin I ; j \in L} \frac{\left[x_{j}-x_{i}+\kappa\right]_{\lambda_{j}}}{\left[x_{j}-x_{i}+\delta\right]_{\lambda_{j}}} \\
& \cdot \prod_{i \in I ; j \in I} \frac{\left[x_{j}-x_{i}+\kappa\right] \lambda_{j}-1}{\left[x_{j}-x_{i}+\delta\right]_{\lambda_{j}-1}} \prod_{i \in I ; j \in L \backslash I} \frac{\left[x_{j}-x_{i}+\kappa-\delta\right]_{\lambda_{j}}}{\left[x_{j}-x_{i}\right]_{\lambda_{j}}}
\end{aligned}
$$




$$
\begin{aligned}
= & \frac{\Delta(x+\lambda \delta)}{\Delta(x)} \prod_{i \in\{1, \ldots, n\} ; j \in L} \frac{\left[x_{j}-x_{i}+\kappa\right] \lambda_{j}}{\left[x_{j}-x_{i}+\delta\right]_{j}} \\
& \cdot \prod_{i \in I ; j \in L \backslash I} \frac{\left[x_{j}-x_{i}+\kappa-\delta\right]}{\left[x_{j}-x_{i}\right]} \prod_{i \in I ; j \in L} \frac{\left[x_{j}-x_{i}+\lambda_{i} \delta\right]}{\left[x_{j}-x_{i}+\kappa+\left(\lambda_{j}-1\right) \delta\right]} .
\end{aligned}
$$

Hence, (2.7) is equivalent to

$$
\begin{gathered}
\sum_{I \subseteq L}(-1)^{|I|}[|I| \kappa+(|\lambda|-|I|) \delta] \prod_{i \in I ; j \in L \backslash I} \frac{\left[x_{j}-x_{i}+\kappa-\delta\right]}{\left[x_{j}-x_{i}\right]} \\
\prod_{i \in I ; j \in L} \frac{\left[x_{j}-x_{i}+\lambda_{j} \delta\right]}{\left[x_{j}-x_{i}+\kappa+\left(\lambda_{j}-1\right) \delta\right]}=0
\end{gathered}
$$

for any $L \neq \phi$ and $\lambda \in \mathbb{N}^{n}$ with $\operatorname{supp}(\lambda)=L$. Setting $\lambda=\epsilon_{L}+v$, we rewrite this in the form

$$
\begin{aligned}
& \sum_{I \subseteq L}(-1)^{|I|}[|I| \kappa+(|v|+|L|-|I|) \delta] \prod_{i \in I ; j \in L \backslash I} \frac{\left[x_{j}-x_{i}+\kappa-\delta\right]}{\left[x_{j}-x_{i}\right]} \\
& \prod_{i \in I ; j \in L} \frac{\left[x_{j}-x_{i}+\delta+v_{j} \delta\right]}{\left[x_{j}-x_{i}+\kappa+v_{j} \delta\right]}=0
\end{aligned}
$$

for any $v \in \mathbb{N}^{n}$ with $\operatorname{supp}(v) \subseteq L$. Since this formula contains only those variables $x_{i}$ with $i \in L$, we have only to consider the case where $L=\{1, \ldots, n\}(n \geq 1)$ :

$$
\begin{aligned}
& \sum_{I \subseteq\{1, \ldots, n\}}(-1)^{|I|}[|I| \kappa+(|v|+n-|I|) \delta] \\
& \quad \cdot \prod_{i \in I ; j \notin I} \frac{\left[x_{j}-x_{i}+\kappa-\delta\right]}{\left[x_{j}-x_{i}\right]} \prod_{i \in I ; j \in\{1, \ldots, n\}} \frac{\left[x_{j}-x_{i}+\delta+v_{j} \delta\right]}{\left[x_{j}-x_{i}+\kappa+v_{j} \delta\right]}=0
\end{aligned}
$$

for any $v \in \mathbb{N}^{n}$. This identity follows from the following functional identity by the change of variables

$$
z_{i}=x_{i}, \quad w_{i}=x_{i}+\delta+v_{i} \delta \quad(i=1, \ldots, n) ; \quad a=\kappa-\delta .
$$

Lemma 2.3 Given two sets of variables $z=\left(z_{1}, \ldots, z_{n}\right), w=\left(w_{1}, \ldots, w_{n}\right)$ and a parameter $a$, the following identity holds as a meromorphic function in $\left(z_{1}, \ldots, z_{n}, w_{1}, \ldots, w_{n}\right)$ for $n \geq 1$ :

$$
\sum_{I \subseteq\{1, \ldots, n\}}(-1)^{|I|} \frac{[|w|-|z|+|I| a]}{[|w|-|z|]} \prod_{i \in I ; j \notin I} \frac{\left[z_{j}-z_{i}+a\right]}{\left[z_{j}-z_{i}\right]} \prod_{i \in I ; k \in\{1, \ldots, n\}} \frac{\left[w_{k}-z_{i}\right]}{\left[w_{k}-z_{i}+a\right]}=0
$$


where $|z|=\sum_{i=1}^{n} z_{i},|w|=\sum_{k=1}^{n} w_{k}$.

Proof We give a proof of the functional identity (2.15) for the case where $[z]=\sigma(z ; \Omega)$ is the Weierstrass sigma function associated with a period lattice $\Omega$. By the classification of $[z]$, it is not difficult to derive (2.15) for any $[z]$ in this class from the case of $\sigma(z ; \Omega)$, by the limiting procedures from $\sigma(z ; \Omega)$ to $\sin (\pi z / \omega)$ and $z$, and by the invariance of (2.15) under the transformation $[z] \rightarrow e^{c z^{2}}[z]$.

Identity (2.15) for $[z]=\sigma(z ; \Omega)$ can be proved by the induction on $n$. Since it holds trivially for $n=1$, we assume $n \geq 2$. We regard the left-hand side of (2.15) as a meromorphic function of $w_{n}$ and denote it by $F\left(w_{n}\right)$ assuming that the other variables are generic. Note first that $F\left(w_{n}\right)$ is an elliptic function possibly with simple poles at $w_{n} \equiv z_{1}-a, \ldots, z_{n}-a$ and $w_{n} \equiv|z|-\left|w^{\prime}\right|$ modulo the period lattice $\Omega$, where $w^{\prime}=\left(w_{1}, \ldots, w_{n-1}\right)$. We first compute the residue of $F\left(w_{n}\right)$ at $w_{n}=z_{n}-a$. Nontrivial residues possibly arise from the terms corresponding to $I$ containing $n$; we parametrize such $I$ 's as $I=J \cup\{n\}$ with $J \subseteq\{1, \ldots, n-1\}$. Then, we have

$$
\begin{aligned}
& \operatorname{Res}_{w_{n}=z_{n}-a}\left(F\left(w_{n}\right) d w_{n}\right) \\
& =\frac{[a]\left[\left|w^{\prime}\right|-\left|z^{\prime}\right|\right]}{\left[\left|w^{\prime}\right|-\left|z^{\prime}\right|-a\right]} \prod_{j \in\{1, \ldots, n-1\}} \frac{\left[z_{j}-z_{n}+a\right]}{\left[z_{j}-z_{n}\right]} \prod_{k \in\{1, \ldots, n-1\}} \frac{\left[w_{k}-z_{n}\right]}{\left[w_{k}-z_{n}+a\right]} \\
& \quad \sum_{J \subseteq\{1, \ldots, n-1\}}(-1)^{|J|} \frac{\left[\left|w^{\prime}\right|-\left|z^{\prime}\right|+|J| a\right]}{\left[\left|w^{\prime}\right|-\left|z^{\prime}\right|\right]} \\
& \quad \prod_{i \in J ; j \in\{1, \ldots, n-1\} \backslash J} \frac{\left[z_{j}-z_{i}+a\right]}{\left[z_{j}-z_{i}\right]} \\
& =0
\end{aligned}
$$

by the induction hypothesis $\left(z^{\prime}=\left(z_{1}, \ldots, z_{n-1}\right)\right)$. Since $F\left(w_{n}\right)$ is symmetric with respect to $\left(z_{1}, \ldots, z_{n}\right)$, we see that $w_{n} \equiv z_{1}-a, \ldots, z_{n}-a$ are all removable poles of $F\left(w_{n}\right)$. Hence, $F\left(w_{n}\right)$ has at most one simple pole in each fundamental parallelogram, which is impossible unless $F\left(w_{n}\right)$ is a constant function since it is an elliptic function. We next look at the value of $F\left(w_{n}\right)$ at $w_{n}=z_{n}$. It is computed as

$$
\begin{aligned}
F\left(z_{n}\right)= & \sum_{I \subseteq\{1, \ldots, n-1\}}(-1)^{|I|} \frac{\left[\left|w^{\prime}\right|-\left|z^{\prime}\right|+|I| a\right]}{\left[\left|w^{\prime}\right|-\left|z^{\prime}\right|\right]} \\
& \cdot \prod_{i \in\{1, \ldots, n-1\} \backslash I ; j \in I} \frac{\left[z_{j}-z_{i}+a\right]}{\left[z_{j}-z_{i}\right]} \prod_{i \in I ; k \in\{1, \ldots, n-1\}} \frac{\left[w_{k}-z_{i}\right]}{\left[w_{k}-z_{i}+a\right]} \\
= & 0
\end{aligned}
$$

again by the induction hypothesis. This implies that $F\left(w_{n}\right)$ is identically zero as a meromorphic function of $w_{n}$. 
Remark 2.4 Lemma 2.3 can be proved in a different way if we make use of the argument of [5]. Recall that in (1.14) of Sect. 1, [5], the following identity is derived from the determinant formula of Frobenius:

$$
\begin{aligned}
& \frac{E\left(T_{z} ; u\right) D(z ; w)}{D(z ; w)} \\
& =\sum_{I \subseteq\{1, \ldots, n\}} u^{|I|} \frac{[\lambda+|z|+|w|+|I| \delta]}{[\lambda+|z|+|w|]} \prod_{i \in I ; j \notin I} \frac{\left[z_{i}-z_{j}+\delta\right]}{\left[z_{i}-z_{j}\right]} \prod_{i \in I ; j \in\{1, \ldots, n\}} \frac{\left[z_{i}+w_{k}\right]}{\left[z_{i}+w_{k}+\delta\right]},
\end{aligned}
$$

where $D(z ; w)$ is the Frobenius determinant

$$
D(z ; w)=\operatorname{det}\left(\frac{\left[\lambda+z_{i}+w_{j}\right]}{[\lambda]\left[z_{i}+w_{j}\right]}\right)_{i, j=1}^{n}=\frac{[\lambda+|z|+|w|] \Delta(z) \Delta(w)}{[\lambda] \prod_{i, j=1}^{n}\left[z_{i}+w_{j}\right]}
$$

and $E\left(T_{z} ; u\right)=\prod_{i=1}^{n}\left(1+u T_{z_{i}}^{\delta}\right)$. This means that

$$
\begin{aligned}
& \frac{[\lambda] \prod_{i, j=1}^{n}\left[z_{i}+w_{j}\right]}{[\lambda+|z|+|w|] \Delta(z) \Delta(w)} \operatorname{det}\left(\frac{\left[\lambda+z_{i}+w_{j}\right]}{[\lambda]\left[z_{i}+w_{j}\right]}+u \frac{\left[\lambda+z_{i}+w_{j}+\delta\right]}{[\lambda]\left[z_{i}+w_{j}+\delta\right]}\right)_{i, j=1}^{n} \\
& =\sum_{I \subseteq\{1, \ldots, n\}} u^{|I|} \frac{[\lambda+|z|+|w|+|I| \delta]}{[\lambda+|z|+|w|]} \prod_{i \in I ; j \notin I} \frac{\left[z_{i}-z_{j}+\delta\right]}{\left[z_{i}-z_{j}\right]} \prod_{i \in I ; j \in\{1, \ldots, n\}} \frac{\left[z_{i}+w_{k}\right]}{\left[z_{i}+w_{k}+\delta\right]} .
\end{aligned}
$$

By setting $u=-1$, we obtain

$$
\begin{aligned}
& \frac{[\lambda] \prod_{i, j=1}^{n}\left[z_{i}+w_{j}\right]}{[\lambda+|z|+|w|] \Delta(z) \Delta(w)} \operatorname{det}\left(\frac{1}{[\lambda]}\left(\frac{\left[\lambda+z_{i}+w_{j}\right]}{\left[z_{i}+w_{j}\right]}-\frac{\left[\lambda+z_{i}+w_{j}+\delta\right]}{\left[z_{i}+w_{j}+\delta\right]}\right)\right)_{i, j=1}^{n} \\
& =\sum_{I \subseteq\{1, \ldots, n\}}(-1)^{|I|} \frac{[\lambda+|z|+|w|+|I| \delta]}{[\lambda+|z|+|w|]} \prod_{i \in I ; j \notin I} \frac{\left[z_{i}-z_{j}+\delta\right]}{\left[z_{i}-z_{j}\right]} \prod_{i \in I ; j \in\{1, \ldots, n\}} \frac{\left[z_{i}+w_{k}\right]}{\left[z_{i}+w_{k}+\delta\right]} .
\end{aligned}
$$

Note that in the limit $\lambda \rightarrow 0$, each entry of the matrix of the left-hand side has a finite limit

$$
\lim _{\lambda \rightarrow 0} \frac{1}{[\lambda]}\left(\frac{\left[\lambda+z_{i}+w_{j}\right]}{\left[z_{i}+w_{j}\right]}-\frac{\left[\lambda+z_{i}+w_{j}+\delta\right]}{\left[z_{i}+w_{j}+\delta\right]}\right)=\frac{\left[z_{i}+w_{j}\right]^{\prime}}{\left[z_{i}+w_{j}\right]}-\frac{\left[z_{i}+w_{j}+\delta\right]^{\prime}}{\left[z_{i}+w_{j}+\delta\right]}
$$

Hence, the left-hand side converges to zero as $\lambda \rightarrow 0$. This implies that

$$
\sum_{I \subseteq\{1, \ldots, n\}}(-1)^{|I|} \frac{[|z|+|w|+|I| \delta]}{[|z|+|w|]} \prod_{i \in I ; j \notin I} \frac{\left[z_{i}-z_{j}+\delta\right]}{\left[z_{i}-z_{j}\right]} \prod_{i \in I ; j \in\{1, \ldots, n\}} \frac{\left[z_{i}+w_{k}\right]}{\left[z_{i}+w_{k}+\delta\right]}=0 .
$$

By replacing each $z_{i}$ with $-z_{i}$, and $\delta$ with $a$, we obtain Lemma 2.3. 


\section{Explicit relations between the two commuting families}

Setting

$$
D_{r}^{(l)}=\frac{[r \kappa+(l-r) \delta]}{[l \delta]} D_{r} \quad(0 \leq r \leq l)
$$

we rewrite the recurrence formula of Theorem 2.1 as

$$
(-1)^{l} H_{l}+(-1)^{l-1} D_{1}^{(l)} H_{l-1}+\cdots-D_{l-1}^{(l)} H_{1}=-D_{l}^{(l)} \quad(l=1,2, \ldots) .
$$

In the matrix form, this means that

$$
\left[\begin{array}{ccccc}
1 & 0 & 0 & \ldots & 0 \\
D_{1}^{(2)} & 1 & 0 & \ldots & \vdots \\
D_{2}^{(3)} & D_{1}^{(3)} & 1 & & \\
\vdots & \vdots & & \ddots & 0 \\
D_{l-1}^{(l)} & D_{l-2}^{(l)} & \ldots & D_{1}^{(l)} & 1
\end{array}\right]\left[\begin{array}{c}
-H_{1} \\
H_{2} \\
-H_{3} \\
\vdots \\
(-1)^{l} H_{l}
\end{array}\right]=-\left[\begin{array}{c}
D_{1}^{(1)} \\
D_{2}^{(2)} \\
D_{3}^{(3)} \\
\vdots \\
D_{l}^{(l)}
\end{array}\right]
$$

Hence, by Cramer's formula we obtain

$$
H_{l}=\operatorname{det}\left[\begin{array}{ccccc}
D_{1}^{(1)} & 1 & 0 & \ldots & 0 \\
D_{2}^{(2)} & D_{1}^{(2)} & 1 & & \vdots \\
\vdots & D_{2}^{(3)} & D_{1}^{(3)} & \ddots & \vdots \\
\vdots & \vdots & \ddots & \ddots & 1 \\
D_{l}^{(1)} & D_{l-1}^{(l)} & \ldots & \ldots & D_{1}^{(l)}
\end{array}\right]=\operatorname{det}\left(D_{i-j+1}^{(i)}\right)_{i, j=1}^{l} \quad(l=1,2, \ldots)
$$

Namely, we have

$$
H_{l}=\operatorname{det}\left(\frac{[(i-j+1) \kappa+(j-1) \delta]}{[i \delta]} D_{i-j+1}\right)_{i, j=1}^{l} \quad(l=1,2 \ldots)
$$

By symmetry, we also have

$$
D_{l}=\operatorname{det}\left(\frac{[(i-j+1) \delta+(j-1) \kappa]}{[i \kappa]} H_{i-j+1}\right)_{i, j=1}^{l} \quad(l=1,2 \ldots)
$$

The recurrence formula (2.1) can also be written as

$$
H_{l}=D_{1}^{(l)} H_{l-1}-\cdots+(-1)^{l-2} D_{l-1}^{(l)} H_{1}+(-1)^{l-1} D_{l}^{(l)} \quad(l=1,2, \ldots) .
$$


Applying this formula repeatedly, we obtain

$$
\begin{aligned}
H_{l} & =\sum_{d=1}^{l}(-1)^{l-d} \sum_{0=l_{0}<l_{1}<\cdots<l_{d}=l} D_{l_{d}-l_{d-1}}^{\left(l_{d}\right)} D_{l_{d-1}-l_{d-2}}^{\left(l_{d-1}\right)} \cdots D_{l_{1}-l_{0}}^{\left(l_{1}\right)} \\
& =\sum_{d=1}^{l}(-1)^{l-d} \sum_{r_{1}+\cdots+r_{d}=l ; r_{i}>0} \prod_{i=1}^{d} D_{r_{i}}^{\left(r_{1}+\cdots+r_{i}\right)} \\
& =\sum_{d=1}^{l}(-1)^{l-d} \sum_{r_{1}+\cdots+r_{d}=l ; r_{i}>0}\left(\prod_{i=1}^{d} \frac{\left[\left(r_{1}+\cdots+r_{i-1}\right) \delta+r_{i} \kappa\right]}{\left[\left(r_{1}+\cdots+r_{i}\right) \delta\right]}\right) D_{r_{1}} \cdots D_{r_{d}} .
\end{aligned}
$$

\section{Kernel identities}

We recall from [5] the duality transformation formula for multiple elliptic hypergeometric series (of type $A$ ): Under the balancing condition $a_{1}+\cdots+a_{m}=b_{1}+\cdots+b_{n}$,

$$
\begin{aligned}
& \sum_{\mu \in \mathbb{N}^{m} ;|\mu|=r} \frac{\Delta(x+\mu \delta)}{\Delta(x)} \prod_{i, j=1}^{m} \frac{\left[x_{i}-x_{j}+a_{j}\right]_{\mu_{i}}}{\left[x_{i}-x_{j}+\delta\right]_{\mu_{i}}} \prod_{i=1}^{m} \prod_{k=1}^{n} \frac{\left[x_{i}+y_{k}-b_{k}\right]_{\mu_{i}}}{\left[x_{i}+y_{k}\right]_{\mu_{i}}} \\
= & \sum_{\nu \in \mathbb{N}^{n} ;|v|=r} \frac{\Delta(y+v \delta)}{\Delta(y)} \prod_{k, l=1}^{n} \frac{\left[y_{k}-y_{l}+b_{l}\right]_{v_{k}}}{\left[y_{k}-y_{l}+\delta\right]_{v_{k}}} \prod_{k=1}^{n} \prod_{i=1}^{m} \frac{\left[y_{k}+x_{i}-a_{i}\right]_{\nu_{k}}}{\left[y_{k}+x_{i}\right]_{v_{k}}} \quad(r=0,1,2, \ldots),
\end{aligned}
$$

where $x=\left(x_{1}, \ldots, x_{m}\right)$ and $y=\left(y_{1}, \ldots, y_{n}\right)$. As we already remarked, when $m=n$ and $a_{i}=b_{i}=\kappa(i=1, \ldots, n)$, this implies

$$
H_{r}^{x} \Phi(x ; y)=H_{r}^{y} \Phi(x ; y) \quad(r=0,1,2, \ldots) .
$$

Let $a_{1}=\cdots=a_{m}=\kappa, b_{1}=\cdots=b_{n}=-\delta$. Then, this transformation formula implies that under the condition $m \kappa+n \delta=0$,

$$
\begin{aligned}
& \sum_{\mu \in \mathbb{N}^{m} ;|\mu|=r} \frac{\Delta(x+\mu \delta)}{\Delta(x)} \prod_{i, j=1}^{m} \frac{\left[x_{i}-x_{j}+\kappa\right]_{\mu_{i}}}{\left[x_{i}-x_{j}+\delta\right]_{\mu_{i}}} \prod_{i=1}^{m} \prod_{k=1}^{n} \frac{\left[x_{i}+y_{k}+\mu_{i} \delta\right]}{\left[x_{i}+y_{k}\right]} \\
= & (-1)^{r} \sum_{K \subseteq\{1, \ldots, n\} ;|K|=r} \prod_{k \in K, l \notin K} \frac{\left[y_{k}-y_{l}-\delta\right]}{\left[y_{k}-y_{l}\right]} \prod_{k \in K i=1}^{m} \frac{\left[y_{k}+x_{i}-k_{i}\right]}{\left[y_{k}+x_{i}\right]} \quad(r=0,1,2, \ldots) .
\end{aligned}
$$

By replacing $y_{k}$ by $-y_{k}$ for $k=1, \ldots, n$, we obtain

$$
\sum_{\mu \in \mathbb{N}^{m} ;|\mu|=r} \frac{\Delta(x+\mu \delta)}{\Delta(x)} \prod_{i, j=1}^{m} \frac{\left[x_{i}-x_{j}+\kappa\right]_{\mu_{i}}}{\left[x_{i}-x_{j}+\delta\right]_{\mu_{i}}} \prod_{i=1}^{m} \prod_{k=1}^{n} \frac{\left[x_{i}-y_{k}+\mu_{i} \delta\right]}{\left[x_{i}-y_{k}\right]}
$$




$$
=(-1)^{r} \sum_{K \subseteq\{1, \ldots, n\} ;|K|=r} \prod_{k \in K, l \notin K} \frac{\left[y_{k}-y_{l}+\delta\right]}{\left[y_{k}-y_{l}\right]} \prod_{k \in K i=1}^{m} \frac{\left[x_{i}-y_{k}-\kappa\right]}{\left[x_{i}-y_{k}\right]} \quad(r=0,1,2, \ldots) .
$$

This means that the dual Cauchy kernel

$$
\Psi(x ; y)=\prod_{i=1}^{m} \prod_{k=1}^{n}\left[x_{i}-y_{k}\right]
$$

satisfies

$$
H_{r}^{(x ; \delta, \kappa)} \Psi(x ; y)=(-1)^{r} D_{r}^{(y ; \kappa, \delta)} \Psi(x ; y) \quad(r=0,1,2, \ldots)
$$

under the condition $m \kappa+n \delta=0$.

\section{The trigonometric cases}

In this section, we consider the trigonometric cases where $[x]=e(x / 2)-e(-x / 2)$ in the notation $e(u)=\exp (2 \pi \sqrt{-1} u)$ of the exponential function. Instead of the parameter $\delta, \kappa \in \mathbb{C}$, we use the multiplicative parameters $q=e(\delta)$ and $t=e(\kappa)$ assuming that $\operatorname{Im}(\delta)>0$ so that $|q|<1$. Note that when $z=e(x)$, we have $[x]=$ $z^{\frac{1}{2}}-z^{-\frac{1}{2}}=-z^{-\frac{1}{2}}(1-z)$, and hence,

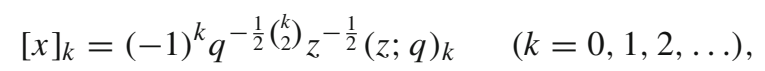

in the standard notation $(z ; q)_{k}=(1-z)(1-q z) \cdots\left(1-q^{k-1} z\right)$ of $q$-shifted factorials.

We denote by $z=\left(z_{1}, \ldots, z_{n}\right)$ the multiplicative variables defined by $z_{i}=e\left(x_{i}\right)$ $(i=1, \ldots, n)$ corresponding to $x=\left(x_{1}, \ldots, x_{n}\right)$. For these $z$ variables, we denote by $T_{q, z_{i}}$ the $q$-shift operator with respect to $z_{i}(i=1, \ldots, n)$ and set $T_{q, z}^{\mu}=T_{q, z_{1}}^{\mu_{1}} \cdots T_{q, z_{n}}^{\mu_{n}}$ for each multi-index $\mu=\left(\mu_{1}, \ldots, \mu_{n}\right) \in \mathbb{N}^{n}$. In this multiplicative notation, it is convenient to introduce the $q$-difference operators $\mathcal{D}_{r}^{z}$ and $\mathcal{H}_{l}^{z}$ normalized so that

$$
D_{r}^{x}=t^{-\frac{1}{2} r(n-r)} \mathcal{D}_{r}^{z} \quad(r=0,1, \ldots, n), \quad H_{l}^{x}=q^{-\frac{1}{2} l} t^{-\frac{1}{2} l n} \mathcal{H}_{l}^{z} \quad(l=0,1,2, \ldots) .
$$

These $q$-difference operators are given explicitly by

$$
\mathcal{D}_{r}^{z}=t^{\left(\begin{array}{c}
r \\
2
\end{array}\right)} \sum_{I \subseteq\{1, \ldots, n\} ;|I|=r} \prod_{i \in I ; j \notin I} \frac{t z_{i}-z_{j}}{z_{i}-z_{j}} T_{q, z}^{\epsilon_{I}}
$$

and

$$
\mathcal{H}_{l}^{z}=\sum_{\mu \in \mathbb{N}^{n} ;|\mu|=l} \prod_{1 \leq i<j \leq n} \frac{q^{\mu_{i}} z_{i}-q^{\mu_{j}} z_{j}}{z_{i}-z_{j}} \prod_{1 \leq i, j \leq n} \frac{\left(t z_{i} / z_{j} ; q\right) \mu_{i}}{\left(q z_{i} / z_{j} ; q\right)_{\mu_{i}}} T_{q, x}^{\mu} .
$$


The recurrence relation (2.1) of Wronski type is then rewritten as follows:

$$
\sum_{r+s=l}(-1)^{r}\left(1-t^{r} q^{s}\right) \mathcal{D}_{r}^{z} \mathcal{H}_{s}^{z}=0 \quad(l=1,2, \ldots)
$$

It is known by [7] that the commuting family of $q$-difference operators $\mathcal{D}_{r}^{z}(r=$ $0,1, \ldots, n)$ act on the ring $\mathbb{C}[z]^{\mathfrak{S}_{n}}=\mathbb{C}\left[z_{1}, \ldots, z_{n}\right]^{\mathfrak{S}_{n}}$ of symmetric polynomials in $z=\left(z_{1}, \ldots, z_{n}\right)$ and that they are simultaneously diagonalized by the (monic) Macdonald polynomials $P_{\lambda}(z)=P_{\lambda}(z \mid q, t)$ indexed by partitions $\lambda=\left(\lambda_{1}, \ldots, \lambda_{n}\right)$ with $l(\lambda) \leq n$ :

$$
\mathcal{D}_{r}^{z} P_{\lambda}(z)=P_{\lambda}(z) e_{r}\left(t^{\delta} q^{\lambda}\right) \quad(r=0,1,2, \ldots, n),
$$

where $e_{r}(\xi)$ stands for the elementary symmetric polynomial of degree $r$ for each $r=0,1, \ldots, n$, and $t^{\delta} q^{\lambda}=\left(t^{n-1} q^{\lambda_{1}}, t^{n-2} q^{\lambda_{2}}, \ldots, q^{\lambda_{n}}\right)$. In terms of the generating function

$$
\mathcal{D}^{z}(u)=\sum_{r=0}^{n}(-u)^{r} \mathcal{D}_{r}^{z}=\sum_{I \subseteq\{1, \ldots, n\}} t^{\left(\begin{array}{c}
|I| \\
2
\end{array}\right)} \prod_{i \in I ; j \notin I} \frac{t z_{i}-z_{j}}{z_{i}-z_{j}} T_{q, z}^{\epsilon_{I}},
$$

formula (5.6) is equivalent to

$$
\mathcal{D}^{z}(z) P_{\lambda}(z)=P_{\lambda}(z) \prod_{i=1}^{n}\left(1-u t^{n-i} q^{\lambda_{i}}\right)
$$

Since $\mathcal{H}_{l}^{z} \in \mathbb{C}\left[\mathcal{D}_{1}^{z}, \ldots, \mathcal{D}_{n}^{z}\right]$, the $q$-difference operators $\mathcal{H}_{l}^{z}(l=0,1,2 \ldots)$ satisfy

$$
\mathcal{H}_{l}^{z} P_{\lambda}(z)=P_{\lambda}(z) g_{l}\left(t^{\delta} q^{\lambda}\right) \quad(l=0,1,2, \ldots)
$$

for some symmetric polynomials $g_{l}(\xi) \in \mathbb{C}[\xi]^{\mathfrak{S}_{n}}$. By the Wronski-type formula (5.5), these polynomials are determined by the recurrence relation

$$
\sum_{r+s=0}(-1)^{r}\left(1-t^{r} q^{s}\right) e_{r}(\xi) g_{s}(\xi)=0 \quad(l=1,2, \ldots)
$$

In view of

$$
E(\xi ; u)=\sum_{r=0}^{n}(-u)^{r} e_{r}(\xi)=\prod_{i=1}^{n}\left(1-u \xi_{i}\right)
$$

let us introduce the generating function $G(\xi ; u)=\sum_{l=0}^{\infty} u^{l} g_{l}(\xi)$. Then, the recurrence formula above is equivalent to the functional equation

$$
E(\xi ; u) G(\xi ; u)=E(\xi ; t u) G(\xi ; q u),
$$


namely

$$
G(\xi ; u)=\frac{E(\xi ; t u)}{E(\xi ; u)} G(\xi ; q u)=\left(\prod_{i=1}^{n} \frac{1-t u \xi_{i}}{1-u \xi_{i}}\right) G(\xi ; q u)
$$

Hence, we have

$$
G(\xi ; u)=\sum_{l=0}^{\infty} u^{l} g_{l}(\xi)=\prod_{i=1}^{n} \frac{\left(t u \xi_{i} ; q\right)_{\infty}}{\left(u \xi_{i} ; q\right)_{\infty}}
$$

where $(u ; q)_{\infty}=\prod_{i=0}^{\infty}\left(1-q^{i} u\right)$. This means that

$$
g_{l}(\xi)=\sum_{\nu_{1}+\cdots+v_{n}=l} \frac{(t ; q)_{\nu_{1}} \cdots(t ; q)_{\nu_{n}}}{(q ; q)_{\nu_{1}} \cdots(q ; q)_{\nu_{n}}} \xi_{1}^{\nu_{1}} \cdots \xi_{n}^{\nu_{n}}=\frac{(t ; q)_{l}}{(q ; q)_{l}} P_{(l)}(\xi) \quad(l=0,1,2, \ldots) .
$$

We introduce the generation function

$$
\mathcal{H}^{z}(u)=\sum_{l=0}^{\infty} u^{l} \mathcal{H}_{l}^{z}=\sum_{\mu \in \mathbb{N}^{n}} u^{|\mu|} \prod_{1 \leq i<j \leq n} \frac{q^{\mu_{i}} z_{i}-q^{\mu_{j}} z_{j}}{z_{i}-z_{j}} \prod_{1 \leq i, j \leq n} \frac{\left(t z_{i} / z_{j} ; q\right) \mu_{i}}{\left(q z_{i} / z_{j} ; q\right)_{\mu_{i}}} T_{q, x}^{\mu}
$$

for our $q$-difference operators $\mathcal{H}_{l}^{z}(l=0,1,2, \ldots)$. Then, the argument above implies that

$$
\mathcal{H}^{z}(u) P_{\lambda}(x)=P_{\lambda}(x) \prod_{i=1}^{n} \frac{\left(u t^{n-i+1} q^{\lambda_{i}} ; q\right)_{\infty}}{\left(u t^{n-i} q^{\lambda_{i}} ; q\right)_{\infty}}
$$

for any partition $\lambda=\left(\lambda_{1}, \ldots, \lambda_{n}\right)$ with $l(\lambda) \leq n$. Note also that the recurrence formula of Wronski type is equivalent to

$$
\mathcal{D}^{z}(u) \mathcal{H}^{z}(u)=\mathcal{D}^{z}(t u) \mathcal{H}^{z}(q u) .
$$

Finally, we give comments on the kernel identities for the trigonometric case. Consider two sets of variables $z=\left(z_{1}, \ldots, z_{m}\right)$ and $w=\left(w_{1}, \ldots w_{n}\right)$, assuming that $m \geq n$. The Cauchy-type kernel for this case is given by

$$
\Pi(z ; w)=\prod_{i=1}^{m} \prod_{k=1}^{n} \frac{\left(t z_{i} w_{k} ; q\right)_{\infty}}{\left(z_{i} w_{k} ; q\right)_{\infty}} .
$$

Then, we have the kernel identities

$$
(D D) \quad \mathcal{D}^{z}(u) \Pi(z ; w)=(u ; t)_{m-n} \mathcal{D}^{w}\left(t^{m-n} u\right) \Pi(z ; w)
$$


$(H H)$

$$
\mathcal{H}^{z}(u) \Pi(z ; w)=\frac{\left(t^{m-n} u ; q\right)_{\infty}}{(u ; q)_{\infty}} \mathcal{H}^{w}\left(t^{m-n} u\right) \Pi(z ; w) .
$$

By the kernel function of dual Cauchy type

$$
\Psi(z ; w)=\prod_{i=1}^{m} \prod_{k=1}^{n}\left(z_{i}-w_{k}\right)
$$

the two families of $q$-difference operators are exchanged as follows:

$(H D)$

$$
(u ; q)_{\infty} \mathcal{H}^{z}(u) \Psi(z ; w)=\left(t^{m} q^{n} u ; q\right)_{\infty} \widehat{\mathcal{D}}^{w}(u) \Psi(z ; w)
$$

where $\widehat{\mathcal{D}}^{w}(u)=\mathcal{D}^{(w \mid t, q)}(u)$ denotes the $q$-difference operator obtained from $\mathcal{D}^{w}(u)=$ $\mathcal{D}^{(w \mid q, t)}(u)$ by exchanging $q$ and $t$.

The three kernel identities $(D D),(H H)$ and $(H D)$ are equivalent to certain special cases of Kajihara's Euler transformation formula [4]: For two sets of variables $z=$ $\left(z_{1}, \ldots, z_{m}\right), w=\left(w_{1}, \ldots, w_{n}\right)$ and parameters $a=\left(a_{1}, \ldots, a_{m}\right), b=\left(b_{1}, \ldots, b_{n}\right)$,

$$
\begin{aligned}
& \frac{(u / \alpha ; q)_{\infty}}{(u ; q)_{\infty}} \sum_{\mu \in \mathbb{N}^{m}}(u / \alpha)^{|\mu|} \prod_{1 \leq i<j \leq m} \frac{q^{\mu_{i}} z_{i}-q^{\mu_{j}} z_{j}}{z_{i}-z_{j}} \prod_{i, j=1}^{m} \frac{\left(a_{j} z_{i} / z_{j} ; q\right)_{\mu_{i}}}{\left(q z_{i} / z_{j} ; q\right)_{\mu_{i}}} \\
& \prod_{i=1}^{m} \prod_{l=1}^{n} \frac{\left(z_{i} w_{l} / b_{l} ; q\right)_{\mu_{i}}}{\left(z_{i} w_{l} ; q\right)_{\mu_{i}}} \\
& =\frac{(u / \beta ; q)_{\infty}}{(u ; q)_{\infty}} \sum_{\nu \in \mathbb{N}^{n}}(u / \beta)^{|v|} \prod_{1 \leq k<l \leq n} \frac{q^{v_{k}} w_{k}-q^{v_{l}} w_{l}}{w_{k}-w_{l}} \prod_{k, l=1}^{n} \frac{\left(b_{l} w_{k} / w_{l} ; q\right)_{\nu_{k}}}{\left(q w_{k} / w_{l} ; q\right)_{\nu_{k}}} \\
& \prod_{k=1}^{n} \prod_{j=1}^{m} \frac{\left(w_{k} z_{j} / a_{j} ; q\right)_{\nu_{k}}}{\left(w_{k} z_{j} ; q\right)_{\nu_{k}}},
\end{aligned}
$$

where $\alpha=a_{1} \cdots a_{m}$ and $\beta=b_{1} \cdots b_{n}$. In fact, one can verify directly that these three kernel identities are equivalent to the following special cases of (5.24), respectively:

$$
\begin{aligned}
& (D D): a_{j}=q^{-1}(j=1, \ldots, m), b_{l}=q^{-1}(l=1, \ldots, n), \\
& (H H): a_{j}=t \quad(j=1, \ldots, m), b_{l}=t \quad(l=1, \ldots, n), \\
& (H D): a_{j}=t \quad(j=1, \ldots, m), b_{l}=q^{-1}(l=1, \ldots, n) \text {. }
\end{aligned}
$$

Acknowledgements M.N. is grateful to the Knut and Alice Wallenberg Foundation (Grant Number KAW 2019.0525) for funding his guest professorship at KTH.

Funding Open access funding provided by Royal Institute of Technology.

Open Access This article is licensed under a Creative Commons Attribution 4.0 International License, which permits use, sharing, adaptation, distribution and reproduction in any medium or format, as long as you give appropriate credit to the original author(s) and the source, provide a link to the Creative Commons licence, and indicate if changes were made. The images or other third party material in this article are included in the article's Creative Commons licence, unless indicated otherwise in a credit line to the material. If 
material is not included in the article's Creative Commons licence and your intended use is not permitted by statutory regulation or exceeds the permitted use, you will need to obtain permission directly from the copyright holder. To view a copy of this licence, visit http://creativecommons.org/licenses/by/4.0/.

\section{References}

1. Aitken, A.C.: Determinants and Matrices. Oliver and Boyd, Edinburgh (1939)

2. Borodin, A., Corwin, I., Remenik, D.: A classical limit of Noumi's $q$-integral operator. SIGMA Symmetry Integr. Geom. Methods Appl. 11, Paper 098, 7 (2015)

3. Feigin, B., Hashizume, K., Hoshino, A., Shiraishi, J., Yanagida, K.: A commutative algebra on degenerate $C P^{1}$ and Macdonald polynomials. J. Math. Phys. 50, 095215, 42 pages (2009)

4. Kajihara, Y.: Euler transformation formula for multiple basic hypergeometric series of type $A$ and some applications. Adv. Math. 187, 53-97 (2004)

5. Kajihara, Y., Noumi, M.: Multiple elliptic hypergeometric series. An approach from the Cauchy determinant. Indag. Math. N.S. 14, 395-421 (2003)

6. Komori, Y., Noumi, M., Shiraishi, J.: Kernel functions of difference operators of Ruijsenaars type and their applications. SIGMA 5, 054, 40 pages (2009)

7. Macdonald, I.G.: Symmetric Functions and Hall Polynomials, 2nd edn. Oxford University Press, Oxford (1995)

8. Masuda, Y.: Kernel identities for van Diejen's $q$-difference operators and transformation formulas for multiple basic hypergeometric series. Ramanujan J. 32, 281-314 (2013)

9. Noumi, M., Shiraishi, J.: A direct approach to the bispectral problem for the Ruijsenaars- Macdonald $q$-difference operators. arXiv:1206.5364, 44 pages

10. Rosengren, H.: New transformations for elliptic hypergeometric series on the root system $A_{n}$. Ramanujan J. 12, 155-166 (2006)

11. Ruijsenaars, S.N.M.: Complete integrability of relativistic Calogero-Moser systems and elliptic function identities. Commun. Math. Phys. 110, 191-213 (1987)

12. Ruijsenaars, S.N.M.: Hilbert-Schmidt operators vs. Integrable systems of Elliptic Calogero-Moser type I. The eigenfunction identities, Commun. Math. Phys. 286, 629-657 (2009); II. The $A_{N-1}$ case: First steps, Commun. Math. Phys. 286, 659-680 (2009)

13. Sano, A.: A commuting family of linear difference operators involving elliptic functions as coefficients (in Japanese), Master's Thesis, Department of Mathematics, Kobe University, 2008

14. Turnbull, H.W.: Theory of Equations. Oliver and Boyd, Edinburgh (1939)

15. Umeda, T.: Application of Koszul complex to Wronski relations for $U\left(\mathfrak{g l}_{n}\right)$. Comment. Math. Helv. 78, 663-680 (2003)

Publisher's Note Springer Nature remains neutral with regard to jurisdictional claims in published maps and institutional affiliations. 